\title{
Terapia ocupacional e consultoria colaborativa: uma revisão narrativa da literatura
}

\section{Occupational therapy and collaborative consultation: a narrative review of the literature}

\author{
Andréa Rizzo dos Santos ${ }^{1}$, Sarah De La Libra ${ }^{2}$
}

http://dx.doi.org/10.11606/issn.2238-6149.v27i1p94-99

Santos AR, De La Libra S. Terapia ocupacional e consultoria colaborativa: uma revisão narrativa da literatura. Rev Ter Ocup Univ São Paulo. 2016 jan.-abr.;27(1):94-9.

RESUMO: O objetivo deste estudo foi realizar uma análise reflexiva da literatura científica nacional sobre a atuação da Terapia Ocupacional na educação inclusiva por meio da Consultoria Colaborativa, identificando as dificuldades encontradas na prática, bem como as estratégias utilizadas e os benefícios resultantes. Foi realizado um levantamento de artigos publicados em quatro periódicos online na área de Terapia Ocupacional e Educação Especial, com os seguintes descritores: "Terapia Ocupacional", "Consultoria Colaborativa", "Consultoria Escolar" e "Inclusão Escolar"; quatro artigos foram selecionados e analisados. Como resultado temos: a deficiência na capacitação de recursos humanos e na provisão de recursos materiais como a principal barreira da educação inclusiva; as estratégias utilizadas variam, mas encontros presenciais entre terapeuta ocupacional e equipe escolar são relatados em todos os artigos; a possibilidade de resolução de problemas e o oferecimento de suporte técnico estão entre os benefícios citados. Como conclusão, os estudos apresentam a consultoria colaborativa como um modelo de prestação de serviço promissor, entretanto há a necessidade de desenvolver mais pesquisas sobre o assunto.

DESCRITORES: Terapia ocupacional; Inclusão educacional; Consultores.
Santos AR, De La Libra S. Occupational therapy and collaborative consultation: a narrative review of the literature. Rev Ter Ocup Univ São Paulo. 2016 Jan.-Apr.;27(1):94-9.

ABSTRACT: This study aimed to perform a reflective analysis of the national scientific literature on the role of Occupational Therapy in the inclusive education by Collaborative Consultation, identifying the difficulties found in the practice, as well as the strategies used and the resulting benefits. We conducted a survey of articles published in four online journals in the field of Occupational Therapy and Special Education, with the following keywords: "Occupational therapy", "Collaborative Consultation", "Educational Consultation" and "Educational Inclusion"; four articles were selected and analyzed. As a result we have: deficiency in gathering human resources and providing material resources as the main barrier of inclusive education; the strategies used vary, but meetings between occupational therapist and school staff are reported in all articles; the possibility of solving problems and offering technical support are among the benefits cited. As a conclusion, the studies present the collaborative consultation as a promising service provision model, but there is a need to develop more researches on this topic.

KEYWORDS: Occupational therapy; Mainstreaming (education); Consultants.

1. Profa. Assistente Doutora do Departamento de Fisioterapia e Terapia Ocupacional - Curso de Terapia Ocupacional- Universidade Estadual Paulista "Júlio de Mesquita Filho” (UNESP) Campus de Marília/SP. E-mail: andreagiar@marilia.unesp.br

2. Terapeuta ocupacional com aprimoramento na área de Terapia Ocupacional e Reabilitação Física pela Faculdade de Medicina da USP. E-mail: sarah.delalibra@hotmail.com

Endereço para correspondência: Departamento de Fisioterapia e Terapia Ocupacional (DEFITO) - UNESP. Av. Higyno Muzzi Filho, 737, Cidade Universitária, Marília, SP. CEP: 17525-000. 


\section{APRESENTAÇÃO}

$\mathrm{A}$ partir da década de 1990, a garantia de acesso a todas as pessoas à educação tornou-se um assunto de notável ascensão no meio acadêmico e político. A Conferência Mundial sobre Educação para Todos (Tailândia, 1990), relembrou "que a educação é um direito fundamental de todos, mulheres e homens, de todas as idades, no mundo inteiro"1.

Com base neste pressuposto, a Conferência Mundial sobre Necessidades Educativas Especiais (Espanha, 1994), convocou a comunidade internacional a somar esforços na busca pela melhoria do acesso à educação para aqueles que apresentam necessidades educacionais especiais (o termo "necessidades educacionais especiais" é utilizado para designar crianças ou jovens cujas necessidades educacionais especiais se originam em função de deficiências ou dificuldades de aprendizagem). $\mathrm{O}$ documento diretor, que ficou conhecido como "Declaração de Salamanca", defende a construção de uma educação inclusiva, na qual, as crianças, jovens e adultos com necessidades educacionais especiais participem do sistema regular de ensino (a menos que existam razões significativas para agir de forma diferente). A demanda dirigida aos governos é o "aprimoramento de seus sistemas educacionais no sentido de se tornarem aptos a incluírem todas as crianças, independentemente de suas diferenças ou dificuldades individuais" .

Diante da proposta de uma mudança sistêmica, os desafios apresentados às escolas são inúmeros e multifacetados, o que torna necessário o desenvolvimento e implementação de políticas de apoio contextualizadas.

No sentido de qualificar e articular iniciativas em prol da educação inclusiva, pessoas com diferentes conhecimentos, perspectivas e experiências estabeleceram parcerias para projetar intervenções com objetivos em comum $^{3,4}$. Professores, psicólogos, fonoaudiólogos, educadores físicos, fisioterapeutas e terapeutas ocupacionais, têm atuado neste contexto por meio de um modelo de prestação de serviço, denominado consultoria colaborativa ${ }^{5,6}$.

Além da consultoria colaborativa, a literatura aponta outros dois modelos de prestação de serviços na área da educação inclusiva, o atendimento direto e o monitoramento $0^{3,7}$

$\mathrm{Na}$ consultoria colaborativa, a atuação profissional é regida por uma filosofia cooperativa, na qual os envolvidos trabalham por um mesmo fim, compartilhando metas, decisões, instruções, responsabilidades e resoluções de problemas ${ }^{8}$.

A consultoria colaborativa possui seis elementos norteadores, sendo eles:

(1) é uma ajuda ou processo de resolução de problemas; (2) ocorre entre alguém que recebe ajuda e alguém que dá ajuda e que tem a responsabilidade pelo bem-estar de uma terceira pessoa; (3) é uma relação voluntária; (4) tanto quem dá a ajuda quanto quem recebe compartilha a solução de problemas; (5) a meta é ajudar a resolver um problema de trabalho atual de quem busca a ajuda e (6) quem ajuda se beneficia da relação, de modo que os futuros problemas poderão ser controlados com mais sensibilidade e habilidade ${ }^{9}$.

$\mathrm{Na}$ literatura é possível encontrar variações na classificação de tais elementos norteadores ${ }^{10}$.

O terapeuta ocupacional é um dos profissionais que tem atuado no contexto escolar por meio da consultoria colaborativa $^{11,12,13}$. A ênfase da terapia ocupacional no contexto escolar incide sobre o desempenho ocupacional do estudante, com vistas a identificar os elementos que afetam a sua capacidade de participação integral nas atividades escolares, como por exemplo, componentes de habilidades do aluno, demandas da atividade, espaço físico, mobiliário e rotina. Diante das demandas identificadas, os serviços prestados podem ser direcionados à equipe educacional, aos pais e/ou à comunidade escolar de modo geral $^{14}$.

Os profissionais de terapia ocupacional que atuam por meio deste modelo de serviço, devem se posicionar como facilitadores, elaborando uma estrutura que favoreça não apenas a conscientização dos envolvidos, mas sua instrumentalização, de modo que seja aumentado o que Spinoza ${ }^{15}$ denomina de "Conatus" - termo que pode "ser entendido, em última instância, como uma maneira de o indivíduo se tornar cada vez mais ativo"15.

A partir do exposto, o presente estudo se propõe a realizar uma análise reflexiva da literatura científica sobre a atuação da terapia ocupacional na educação inclusiva brasileira por meio da consultoria colaborativa. Para tanto, adotou-se os princípios metodológicos da revisão narrativa, uma vez que esta técnica permite $o$ aprofundamento nas análises críticas e interpretações sobre um assunto específico, sendo possível averiguar como o mesmo se encontra no momento atual ${ }^{16}$. Trata-se, portanto, de uma tentativa de aprofundar as discussões sobre a temática, refletindo sobre as características da consultoria colaborativa, bem como sua importância. Também serão 
abordadas questões correlacionadas ao tema da inclusão educacional.

\section{DESENVOLVIMENTO}

Foi realizada uma pesquisa bibliográfica em quatro periódicos nacionais online da área de Terapia Ocupacional e Educação Especial (Revista de Terapia Ocupacional da USP; Cadernos de Terapia Ocupacional da UFSCAR; Revista Educação Especial da UFSM e Revista Brasileira de Educação Especial), com os seguintes descritores: "Terapia Ocupacional", "Consultoria Colaborativa", "Consultoria Escolar" e "Inclusão Escolar; sendo selecionados quatro artigos, os quais foram analisados com ênfase nas estratégias de intervenção utilizadas e principais dificuldades e benefícios apontados pelos autores em relação à atuação do terapeuta ocupacional no contexto da educação inclusiva por meio da consultoria colaborativa. A categorização dos dados dos quatro artigos é apresentada no Quadro 1. Os artigos selecionados foram identificados como A1, A2, A3 e A4. No decorrer do texto, procurouse estabelecer um diálogo entre a literatura nacional encontrada e referências teóricas internacionais.

É consenso que apenas a matrícula do aluno não garante sua real inclusão, ou seja, o fato de o indivíduo estar na escola não significa que o mesmo participe das atividades desenvolvidas. Faz-se necessário que seja assegurada a "possibilidade de ingresso e permanência do aluno na escola com sucesso acadêmico", como apontam Glat e Blanco ${ }^{17}$. Ao adentrar nesse universo, muitos autores sinalizaram as dificuldades encontradas no cotidiano da inclusão escolar, envolvendo a permanência do aluno com necessidades educativas especiais na escola e o processo de ensino-aprendizagem.

Ide et al. ${ }^{13}$, em pesquisa realizada com orientadores educacionais identificaram o ambiente escolar, a (falta de) capacitação, apoio especializado e parcerias, como sendo as principais dificuldades com as quais os professores de alunos com necessidades educativas especiais se deparam. Os autores dos quatro artigos analisados, também encontraram elementos desfavoráveis, como barreiras arquitetônicas, escassez de material adaptado, despreparo dos professores e ainda, obstáculos atitudinais detectáveis em demonstrações de piedade ou compadecimento estanques.

Assim, os programas de consultoria colaborativa apresentam-se como uma resposta alternativa a necessidade de desenvolver modelos de prestação de serviço compatíveis com os objetivos e propósitos da educação inclusiva ${ }^{18}$.
Dunn ${ }^{19}$ comparou a consultoria colaborativa com o modelo de atendimento individual em estudo piloto no qual participaram professores e terapeutas ocupacionais, e concluiu que ambos os serviços foram eficazes no que tange a realização dos objetivos; no entanto, os professores relataram interações mais positivas relacionadas à consultoria colaborativa.

Os resultados encontrados por Nochajski ${ }^{18}$, os quais indicam que a consultoria colaborativa resultou em benefícios tanto para os estudantes quanto para os profissionais envolvidos, corroboram com os apontamentos de A1, A2, A3 e A4. Estes autores destacaram que a consultoria colaborativa possibilitou a resolução de problemas que comprometiam a qualidade de aprendizado e a convivência; melhora das sensações de frustração e impotência diante das dificuldades encontradas; promoção de desenvolvimento pessoal e profissional dos educadores, permitindo a conscientização quanto à diversidade e a necessidade de o ambiente facilitar a inclusão das crianças com necessidades educacionais especiais.

Em relação às estratégias utilizadas, os artigos analisados apontaram: discussão de casos, orientação sobre características particulares dos alunos, dinâmicas de grupo, apresentação da tecnologia assistiva e comunicação alternativa, leitura e discussão de textos, oficina de construção de materiais pedagógicos, adaptação de mobiliários, adaptação do currículo pedagógico e estabelecimento de objetivos em comum. Encontros presenciais (mensais ou semanais) entre professores (e outros membros da comunidade escolar) e terapeutas ocupacionais (pesquisadores) também foram utilizados como estratégia em todos os programas.

Embora os artigos consultados não descrevam estes encontros, afirmam que o contato permitiu oportunidades para a discussão das problemáticas que permeiam o cotidiano da educação inclusiva, bem como a construção de propostas de solução, valorizando o trabalho em equipe. Villa e Thousand ${ }^{20}$, consideram fundamental promover um ambiente que favoreça a discussão entre os membros da equipe sobre seus sentimentos em relação à colaboração.

A partir da leitura, foi possível conceber que se bem estruturados e planejados, estes momentos podem proporcionar a apropriação e desvelamento das dificuldades, a decomposição das paixões tristes e a composição das paixões alegres, resultando no aumento do Conatus, tornando-se assim, bons encontros ${ }^{21}$. Portanto, um dos fatores que pode influenciar o sucesso da consultoria colaborativa é a qualidade das reuniões/ encontros e do vínculo estabelecido entre terapeuta e 


\begin{tabular}{|c|c|c|c|c|c|c|}
\hline 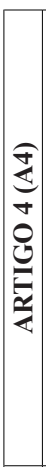 & 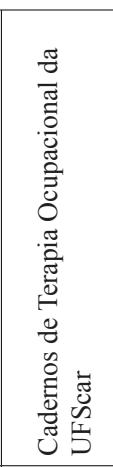 & 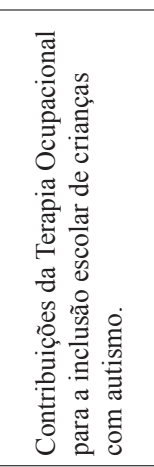 & 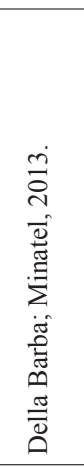 & 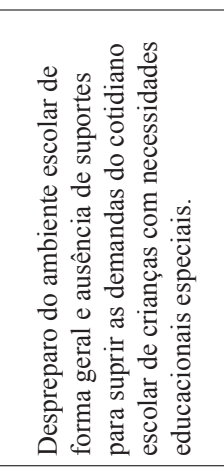 & 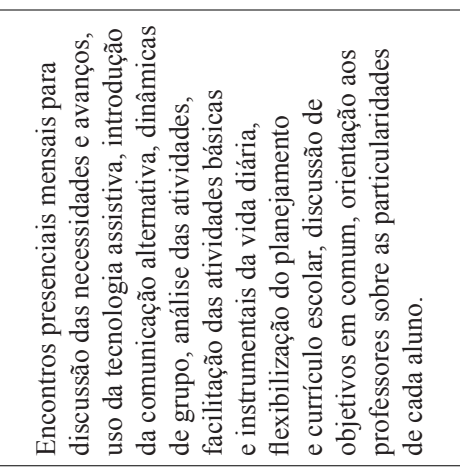 & 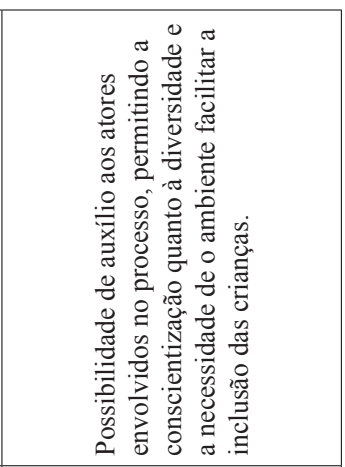 \\
\hline 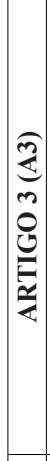 & 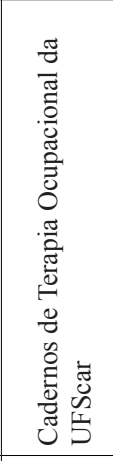 & 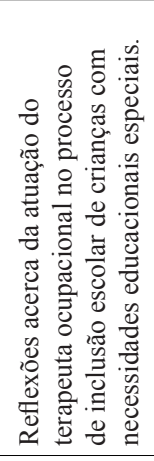 & 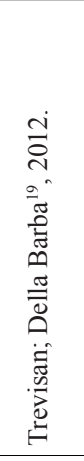 & 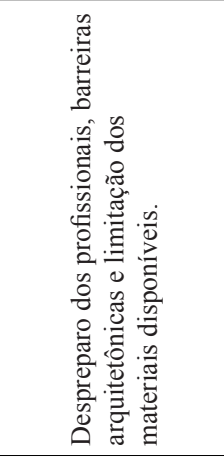 & 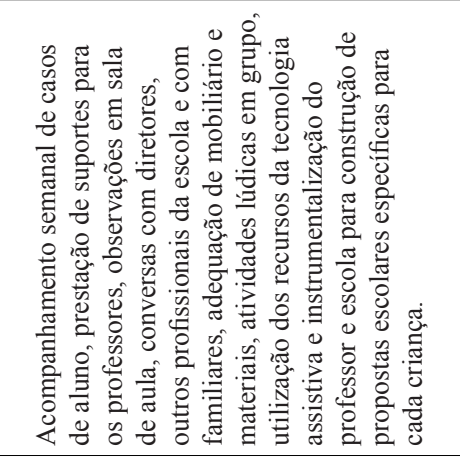 & 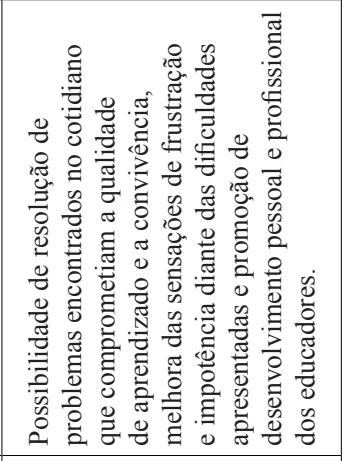 \\
\hline 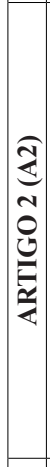 & 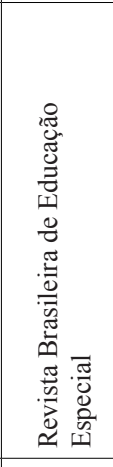 & 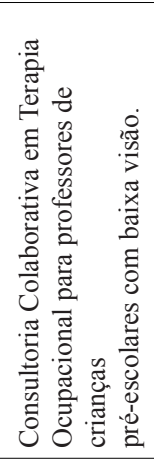 & 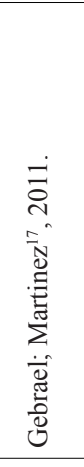 & 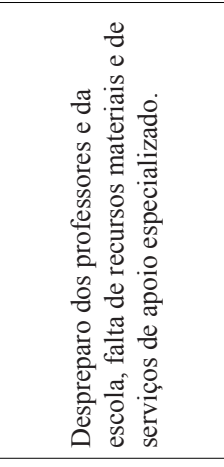 & 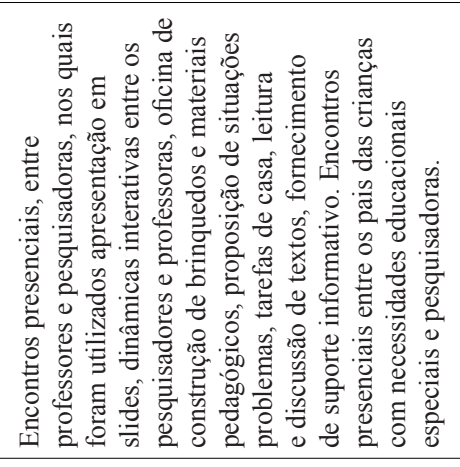 & 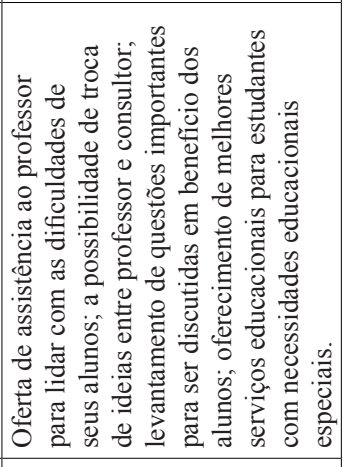 \\
\hline 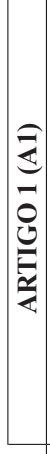 & 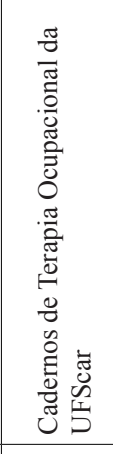 & 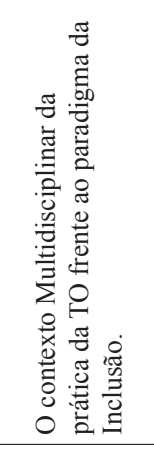 & 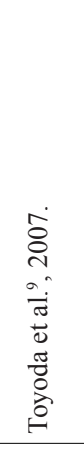 & 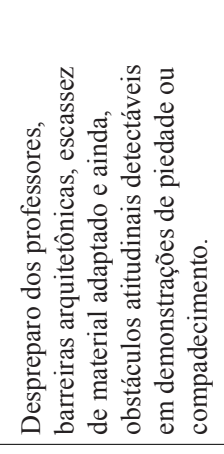 & 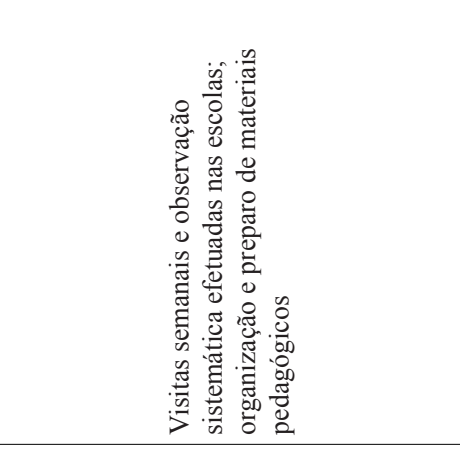 & 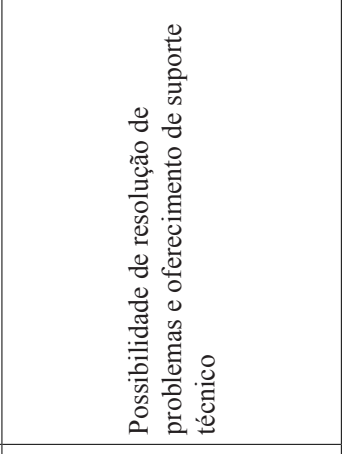 \\
\hline & 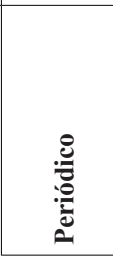 & $\stackrel{\varrho}{\Xi}$ & 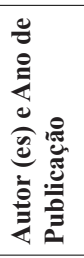 & 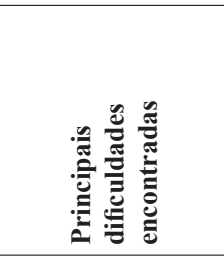 & 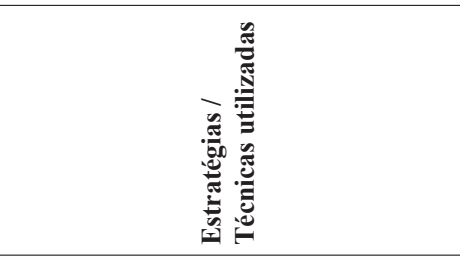 & 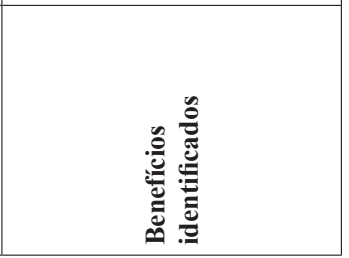 \\
\hline
\end{tabular}


comunidade escolar. Nesse sentido, Bondy e Brownell ${ }^{22}$ afirmam que a qualidade da comunicação/colaboração estabelecida influenciam a eficácia da consultoria.

O terapeuta ocupacional, que é um profissional habilitado para realizar a análise da situação que necessita de intervenção, considerando as diversas áreas e contextos envolvidos, pode favorecer o desenvolvimento de bons encontros por meio da atuação direcionada.

No que diz respeito à consultoria colaborativa, é importante que este profissional analise: o território em que a escola está inserida (população jovem/adulta; bairro empresarial/residencial; condição social; acesso à cultura/lazer; vulnerabilidade às drogas, violência; quem frequenta a escola e o que a escola significa para o entorno); o contexto escolar (Aspectos físicos - espaço grande/ pequeno; higiene; acessibilidade; mobiliários e layout da sala de aula; existência de sala de recursos/multifuncional; rotina; ambientes externos, parque, refeitório; reuniões pedagógicas e etc. Materiais- o que está disponível; quais os mais utilizados; quem financia; possibilidade de adaptação. Recursos-Humanos- equipe escolar; existência de conflitos; sobrecarga de funções; existência de outros serviços de apoio e etc.) e a participação da comunidade nas atividades escolares (como é a relação família $\mathrm{x}$ escola; existência de oportunidades para encontros e diálogos entre a família e a escola e etc) ${ }^{14,21}$.

A identificação das características peculiares sugere um olhar individual para cada ambiente escolar, pois, a partir das informações coletadas por meio de entrevistas e/ou observação, o terapeuta ocupacional pode introduzir no planejamento da consultoria, elementos específicos, condizentes com a realidade do local de trabalho. Assim, esse cuidado pode elevar a probabilidade de sucesso, uma vez que oportuniza a empatia entre os participantes e a configuração do serviço. Consequentemente, essa empatia pode gerar maior envolvimento dos profissionais que veem a possibilidade de terem suas necessidades atendidas ${ }^{23}$.

Mas como mensurar os benefícios? Apenas o artigo A2 relatou a aplicação de testes para investigar os resultados atingidos pelo programa. Este consistia numa entrevista semiestruturada, aplicada no início do programa (pré-teste) e um estudo de caso, aplicado ao final do programa (pós-teste).

Sobre mensuração, Chaves et al. ${ }^{24} \mathrm{em}$ seus estudos afirmaram que a necessidade de sistematizar a mensuração dos resultados obtidos nas intervenções, precisa ser considerada e estimulada, uma vez que favorece o reconhecimento científico da profissão, possibilita a produção de conhecimento específico da área, bem como assegura a confiabilidade das intervenções. Na medida em que a credibilidade do trabalho é assegurada, a divulgação do mesmo acaba por ser um processo natura ${ }^{24}$. Assim, é possível inferir que haja a necessidade de se inserir nos programas de consultoria colaborativa, medidas fidedignas de avaliação do trabalho desenvolvido, a fim de que, a partir da análise dos resultados, seja possível identificar e corrigir as falhas, aumentando a qualidade do serviço e a consolidação científica do mesmo ${ }^{7}$.

Kammis e Dunn ${ }^{3}$, em estudo datado de 1996, apontaram que a consultoria colaborativa era um modelo pouco conhecido pelos terapeutas ocupacionais. Coppede et al. ${ }^{25}$ publicaram em 2014 um levantamento da produção científica nacional sobre as contribuições da Terapia Ocupacional no processo de inclusão escolar e dos 33 artigos selecionados no estudo, apena um discutia a consultoria colaborativa.

Diante desses dados, fica evidente a existência de uma lacuna na produção científica nacional que trata desse assunto, sendo necessário o incentivo ao desenvolvimento de novas pesquisas e planos de ações envolvendo a terapia ocupacional e a consultoria colaborativa ${ }^{18}$.

\section{CONCLUSÃO}

O estudo identificou atuações isoladas de terapeutas ocupacionais em território brasileiro, que adotando a consultoria colaborativa, desenvolvem importantes trabalhos no ambiente escolar e contribuem diretamente para que haja avanços reais no processo de aprimoramento no sistema educacional brasileiro. No entanto, a deficiência na capacitação de recursos humanos e na provisão de recursos materiais se constitui a principal barreira da educação inclusiva. As estratégias utilizadas variam, mas encontros presenciais entre terapeuta ocupacional e equipe escolar são relatados em todos os programas e a qualidade dos mesmos é um fator que precisa ser considerado.

Os estudos afirmam que os resultados da consultoria colaborativa são positivos, maséimportante que os estudantes e profissionais que desenvolvem trabalhos e pesquisas sobre a atuação da terapia ocupacional na consultoria colaborativa, se conscientizem e elaborem medidas para mensurar os benefícios alcançados e compartilhem suas experiências.

A prestação de serviço por meio da consultoria colaborativa demanda estudos mais específicos, a fim de sistematizar as abordagens teóricas utilizadas, aumentando a notoriedade do serviço e a oferta do mesmo. 


\section{REFERÊNCIAS}

1. Declaração Mundial sobre Educação para Todos. Aprovada pela Conferência Mundial sobre Educação para Todos, Jomtien, Tailândia, 5-9 mar. 1990. Satisfação das Necessidades Básicas de Aprendizagem [citado 9 ago. 2014]. Disponível em: http://www.unicef.org/brazil/pt/resources_10230.htm.

2. Declaração de Salamanca. Sobre princípios, políticas e práticas na área das necessidades educativas especiais [citado 9 ago. 2014]. Disponível em: http://portal.mec.gov. br/seesp/arquivos/pdf/salamanca.pdf.

3. Kemmis BL, Dunn W. Collaborative consultation: the efficacy of remedial interventions in school contexts. Occup Ther J Res. 1996;50(9):709-17. doi: 10.5014/ajot.50.9.709.

4. Mendes EG, Almeida MA, Toyoda CY. Inclusão escolar pela via da colaboração entre educação especial e educação regular. Educ Rev. 2011;41:80-93. http://dx.doi. org/10.1590/S0104-40602011000300006

5. Kampwirth TJ. Collaborative consultation in the schools. 2a ed. Upper Saddle River, NJ: Prentice Hall; 2003.

6. Fonseca-Janes CRX, Brito MC, Janes R, organizadores. A construção da educação inclusiva: enfoque muldisciplinar. São Paulo: Cultura Acadêmica; 2012. p.15-28.

7. Swinth YL. Avaliação e tratamento de terapia ocupacional com relação à educação. In: Willard \& Spackman: terapia ocupacional. 11a ed. Rio de Janeiro: Guanabara Koogan; 2011. p.621.

8. Araújo SLS, Almeida MA. Contribuições da consultoria colaborativa para a inclusão de pessoas com deficiência intelectual. Reva Educ Espec. 2014;27(49):341-52. http:// dx.doi.org/ 10.5902/1984686x8639.

9. Persons RD, Meyers J. Developing consultation skills. USA/San Francisco: Jossey Bass; 1984 apud Toyoda CY, et al. O contexto multidisciplinar da prática da terapia ocupacional frente ao paradigma da inclusão escolar. Cad Ter Ocup UFSCar. 2007;15(2):124.

10. Churchley CM. Collaborative consultation in the context of inclusion [dissertation]. 2006. Dissertação de Adeliade, Austr.: Flinders University; 2006. Available from: http:// www.flinders.edu.au/ehl/fms/projects_files/6_2006.pdf.

11. Rocha EF, Luiz A, Zulian MAR. Reflexões sobre as possíveis contribuições da terapia ocupacional nos processos de inclusão escolar. Rev Ter Ocup Univ São Paulo. 2003;14(2):72-8. http://dx.doi.org/10.11606/ issn.2238-6149.v14i2p72-78.

12. Jurdi APS, Brunello MIB, Honda M. Terapia ocupacional e propostas de intervenção na rede pública de ensino. Rev Ter Ocup Univ São Paulo. 2004;15(1):26-32. http://dx.doi. org/10.11606/issn.2238-6149.v15i1p26-32.

13. Ide MG, Yamamoto BT, Silva CCB. Identificando possibilidades de atuação da terapia ocupacional na inclusão escolar. Cad Ter Ocup UFSCar. 2011;19(3):323-32. http:// dx.doi.org/10.4322/cto.2011.004.

14. Munguba MC. Inclusão escolar. In: Cavalcanti A, Galvão C. Terapia ocupacional fundamentação \& prática. Rio de Janeiro: Guanabara Koogan; 2011. p.519-25.

15. Pereira RR. O Conatus de Spinoza: auto-conservação ou liberdade. Cad Espinosanos. 2008(19):74. Disponível em: http://www.revistas.usp.br/espinosanos/article/ view/89343/92215.

16. Rother ET. Revisão sistemática $\mathrm{x}$ revisão narrativa. Acta Paul Enferm. 2007;20(2):5-6. Disponível em: http://www. redalyc.org/articulo.oa?id=307026613004.

17. Glat R, Blanco LMV. Educação especial no contexto de uma educação inclusiva. In: Glat R, organizador. Educação inclusiva: cultura e cotidiano escolar. Rio de Janeiro: 7 Letras; 2007. p.15-35.

18. Nochajski SM. Collaboration between team members in inclusive educational settings. Occup Ther Health Care. 2002;15(3-4):101-12. http://dx.doi.org/10.1080/ J003v15n03_06.

19. Dunn W. A comparison of service provision models in school-based occupational therapy services: a pilor study. Occup Ther J Res. 1990;10:300-20. http://dx.doi. org/10.1177/153944929001000505.

20. Villa RA, Thousand JS. Instilling collaboration for inclusive schooling as a way of doing business in public schools. 1996; 17, 169-182. http://dx.doi.org/10.1177/074193259601700306.

21. Rocha EF. A Terapia ocupacional e as ações na educação: aprofundando interfaces. Rev Ter Ocup Univ São Paulo. 2007;18(3):122-7. http://dx.doi.org/10.11606/issn.22386149.v18i3p122-127.

22. Bondy E, Brownell MT. Overcoming barriers to collaboration among-partners in teaching. Intervention School Clin. 1997;33:112-6. http://dx.doi.org/10.1177/105 345129703300207.

23. Keys SG, Bemak F, Carpenter SL, King-Sears ME. Collaborative consultant: a new role for counselors serving at-risk youth. J Couns Develop. 1998;76:123-33. http:// dx.doi.org/10.1002/j.1556-6676.1998.tb02385.x.

24. Chaves GFS, et al. Escalas de avaliação para Terapia Ocupacional no Brasil. Rev Ter Ocup Univ São Paulo. 2010;21(3):240-246. http://dx.doi.org/10.11606/issn.22386149.v21i3p240-246.

25. Coppede AC, et al. Produção científica da terapia ocupacional na inclusão escolar: interface com a Educação Especial e contribuições para o campo. Rev Educ Espec. 2014;27(49):471-84. http://dx.doi. org/10.5902/1984686X8281. 